\title{
On special limit of non-supersymmetric effective actions of type II string theory
}

\author{
Ehsan Hatefi $^{1,2,3, a}$, Petr Vasko ${ }^{4, b}$ \\ ${ }^{1}$ Faculty of Physics, University of Warsaw, ul. Pasteura 5, 02-093 Warsaw, Poland \\ ${ }^{2}$ Scuola Normale Superiore and INFN, Piazza dei Cavalieri, 7, 56126 Pisa, Italy \\ ${ }^{3}$ Faculty of Mathematics Charles University, Mathematical Institute, 18675 Prague, Czech Republic \\ ${ }^{4}$ Faculty of Physics, University of Warsaw, Warsaw, Poland
}

Received: 31 July 2018 / Accepted: 24 January 2019 / Published online: 12 February 2019

(c) The Author(s) 2019

\begin{abstract}
In this paper we first address four point functions of string amplitudes in both type IIA and IIB string theories. Making use of non-BPS scattering amplitudes, we explore not only several Bianchi identities that hold in both transverse and world volume directions of the brane, but also we reveal various new couplings. These couplings can just be found by taking into account the mixed pull-back and Taylor couplings where their all order alpha-prime higher derivative corrections have been derived as well. For the first time, we also explore the complete form of a six point non-BPS amplitude, involving three open string tachyons, a scalar field and a Ramond-Ramond closed string in both IIA, IIB. In a special limit of the amplitude and using the proper expansion we obtain an infinite number of bulk singularities that are being constructed in the effective field theory. Finally, using new couplings we construct all the other massless and tachyon singularities in type IIA, IIB string theories. All higher derivative corrections to these new couplings to all orders in $\alpha^{\prime}$ and new restricted Bianchi identities have also been obtained.
\end{abstract}

\section{Introduction}

Among several goals of theoretical physicists and in particular string theorists, we may point out two common interests in uncovering more information: about how the supersymmetry gets broken as well as working out new couplings/ interactions on time dependent backgrounds. If we try to deal with non-supersymmetric (unstable) branes, then one may be able to properly address some of the open questions and also might be able to deepen insight in many properties of various different string theories [1-9]. Since the duality transforma-

\footnotetext{
a e-mails: ehsan.hatefi@sns.it; ehsanhatefi@gmail.com

b e-mail: vasko.petr@gmail.com
}

tion is not promising in this context anymore, one needs to be aware of the fact that for non-BPS branes just scattering amplitudes and Conformal Field Theory (CFT) methods [10] would exactly determine corrections to all orders in $\alpha^{\prime}$ of the effective actions of string theory.

Making use of non-supersymmetric branes, the so-called Sakai-Sugimoto model $[11,12]$ and the symmetry breaking for holographic QCD models have become known [13,14]. Tachyons do play a crucial role in the instability of the aforementioned systems so it would be important to consider tachyons and try to obtain their effective actions in both type IIA and IIB string theories and also explore their new couplings in Effective Field Theory (EFT).

The leading order non-BPS effective actions including tachyonic modes were proposed in $[15,16]$, where some of their properties such as their decays and tachyon condensation have also been clarified in detail [17]. Following [8,9], one reveals how to embed non-BPS branes in the effective actions. We studied D-brane-anti-D-brane systems $[19,20]$. Recently, the generalization of effective actions of D-brane-anti-D-brane system to all orders in alpha-prime for both Chern-Simons and Dirac-Born-Infeld (DBI) effective actions was discovered [21]. Another example would be related to tachyon condensation that has been investigated in [22] in detail. For the D-brane-anti-D-brane system, once the distance between brane and anti-brane becomes smaller than the string length scale, two real tachyonic strings would appear. They are related to strings stretched from D-brane to anti-D-brane and vice versa.

Here we would like to deal with $\mathrm{N}$ coincident non-BPS branes and try to embed tachyonic modes and their corrections in EFT. We take the non-BPS scattering amplitude formalism as a theoretical framework or laboratory to discover their effective actions, including their corrections to all order in $\alpha^{\prime}$ in string theory in an efficient and consistent way of 
matching string results with EFT. To deal with the dynamics of unstable branes, we highlight the recent work done by Polchinski et al. [23] where various explanations within the context of the brane 's effective actions through EFT have been discussed. Not only brane production [24-31], but also inflation in string theory in the procedure of KKLT [32-34] can be mentioned. To observe a review of open strings and their features we point out to [35-41]. For reviews see [42, 43]

In this paper we deal with a non-BPS four point function and explore some Bianchi identities and new EFT couplings that come from the mixed pull-back formalism and Taylor expansion and then try to use the lower point functions to exactly build for the first time a non-BPS six point function. Having used the scattering amplitude methods, we would also fix some of the ambiguities of the corrections in string theory and reveal new string couplings in both type II string theories. ${ }^{1}$

One can try to relate some of the new couplings to the AdS/CFT correspondence $[45,46]$. It is also worth making a remark on the D-brane-anti-D-brane systems, as they do affect not only the problem of stability of KKLT model but also string compactifications $[47,48]$ and in particular the socalled large volume scenario. The relation between D-branes and Ramond-Ramond (RR) charges is well established [49], where one could also take into account some brane's bound states [50]. All the EFT methods of deriving the WessZumino (WZ) and DBI effective actions are given in [51,52].

The paper is organized as follows. First we study a four point function including a closed string RR and a transverse scalar field and a real tachyon on the world volume of non-BPS branes; an RR and two tachyons have been fully addressed in [53]. Then we build all order $\alpha^{\prime}$ higher derivative corrections to it and explore a pattern from this calculation to reconstruct all singularity structures of the higher point functions of non-BPS branes.

Our notations for indices are summarized by the following.

$\mu, v=0,1, \ldots, 9$ represent the whole ten dimensional space-time, $a, b, c=0,1, \ldots, p$ show the world volume indices, and finally for transverse directions of the brane $i, j=p+1, \ldots, 9$ are taken accordingly.

We establish a new coupling among RR, the tachyon field living on the world volume of a non-BPS brane and one massless scalar field representing a transverse direction of the brane.

This new mixed WZ-Taylor expansion is given by

$$
\frac{2 i \beta^{\prime} \mu_{p}^{\prime}}{(p) !}\left(2 \pi \alpha^{\prime}\right)^{2} \int_{\Sigma_{p+1}} \epsilon^{a_{0} \ldots a_{p}} C_{i a_{0} \ldots s a_{p-2}} D_{a_{p-1}} T D_{a_{p}} \phi^{i}
$$

\footnotetext{
1 To work with some higher point functions and for their corrections we just highlight [35-40] and [41-44] accordingly.
}

where $\mu_{p}^{\prime}$ is the RR charge of the brane and $\beta^{\prime}$ is the WZ normalization constant.

Note that the integration should be taken on $(p+1)$ world volume directions and in order to cover the whole world volume indices we extract the coupling and write it as (1). We also explore its corrections to all orders in the higher derivatives too.

Having set all lower point functions of the non-BPS branes, we would clarify more hidden symmetries in a nonBPS context. Hence we make use of all the CFT techniques to a six point correlation of an RR, a scalar field and three tachyons. We first find the entire correlators of $<V_{C^{-1}} V_{\phi^{-1}} V_{T^{0}} V_{T^{0}} V_{T^{0}}>$ in type IIA (IIB) and then we just illustrate the final result in a different picture of the scalar field. Basically we explore $<V_{C^{-1}} V_{\phi^{0}} V_{T^{-1}} V_{T^{0}} V_{T^{0}}>$ and argue that using this particular case we would be able to precisely obtain all bulk singularity structures that are not present in the other picture. Using selection rules [54] for the non-BPS amplitudes, EFT and in a particular soft limit, we discover the ultimate answer for the S-matrix. Having set all symmetries of the S-matrix, we explore the expansion of the S-matrix. Using the soft limit we generate not only all the infinite massless singularities, but also an infinite number of u-channel bulk singularity structures can be precisely reconstructed in an EFT and we arrive at a perfect match between the string amplitudes and the EFT counterparts. Finally, we use all the higher derivative corrections of two tachyon-two scalar couplings to be able to produce an infinite number of the scalar field singularities as well. It is worth to emphasize that, since there is no coupling between two tachyons and a scalar field, the amplitude (as can be seen from the ultimate result of the S-matrix) has no singularity in the $t, s, v$ channels at all. The DBI part of the effective action for non-BPS branes is

$$
\begin{aligned}
& S_{\mathrm{DBI}} \sim \int d^{p+1} \sigma \mathrm{STr}\left(V\left(T^{i} T^{i}\right) \sqrt{\left.1+\frac{1}{2}\left[T^{i}, T^{j}\right]\left[T^{j}, T^{i}\right]\right)}\right. \\
& \left.\times \sqrt{-\operatorname{det}\left(\eta_{a b}+2 \pi \alpha^{\prime} F_{a b}+2 \pi \alpha^{\prime} D_{a} T^{i}\left(Q^{-1}\right)^{i j} D_{b} T^{j}\right)}\right),
\end{aligned}
$$

where $V\left(T^{i} T^{i}\right)=\mathrm{e}^{-\pi T^{i} T^{i} / 2}$, and

$Q^{i j}=I \delta^{i j}-i\left[T^{i}, T^{j}\right]$,

$i, j=1,2$, i.e., $T^{1}=T \sigma_{1}, T^{2}=T \sigma_{2}$. The DBI part of the $\mathrm{D}$-brane-anti-D-brane is given in [21]. If we make the kinetic terms symmetrized, find the traces and then use the ordinary trace, the action will get replaced by Sen's action [55]. However, in [56,57] by direct CFT computations and scattering amplitudes we have shown that Sen's effective action does not provide a result consistent with the string amplitudes. The expansion of the S-matrices is consistent with the tachyon's potential $V(|T|)=\mathrm{e}^{\pi \alpha^{\prime} m^{2}|T|^{2}}$, which comes from 
BSFT [58-60]. On the other hand, the WZ action is given by

$$
S_{\mathrm{WZ}}=\mu_{p}^{\prime} \int_{\Sigma_{(p+1)}} C \wedge \operatorname{Str}^{i 2 \pi \alpha^{\prime} \mathcal{F}} .
$$

To consider interactions with tachyons, one can make contact with the super connection of the non-commutative geometry [61-63], where the curvature is

$i \mathcal{F}=\left(\begin{array}{cc}i F-\beta^{\prime 2} T^{2} & \beta^{\prime} \mathrm{DT}, \\ \beta^{\prime} \mathrm{DT} & i F-\beta^{\prime 2} T^{2}\end{array}\right)$

One can find different types of WZ couplings from the above actions to generate a consistent result between the string amplitudes and the EFT, such as

$$
\begin{aligned}
C & \wedge \mathrm{STr} i \mathcal{F}=2 \beta^{\prime} \mu_{p}^{\prime}\left(2 \pi \alpha^{\prime}\right) C_{p} \wedge \mathrm{DT} \\
C & \wedge \mathrm{STr} i \mathcal{F} \wedge i \mathcal{F}=\beta^{\prime} \mu_{p}^{\prime}\left(2 \pi \alpha^{\prime}\right)^{2} \\
& \times\left(C_{p-1} \wedge \mathrm{DT} \wedge(\mathrm{DT})+C_{p-2} \wedge F \wedge \mathrm{DT}\right) .
\end{aligned}
$$

\section{All order $\alpha^{\prime}$ corrections to $<V_{C^{-2}} V_{T^{0}} V_{\phi^{0}}>$}

In order to actually address the entire form of a four point function of an RR, a real tachyon and a scalar field in both type IIA and IIB string theories, one must apply conformal field theory methods to the complete S-matrix elements and explore whether or not there are some bulk singularity structures and also one should see how one might be able to find all order contact interactions. To obtain all the correlation functions, one needs to know the vertex operators where their complete forms are shown by

$$
\begin{aligned}
& V_{T}^{(0)}(x)=\alpha^{\prime} i k_{1} \cdot \psi(x) \mathrm{e}^{\alpha^{\prime} i k_{1} \cdot X(x)} \lambda \otimes \sigma_{1}, \\
& V_{T}^{(-1)}(x)=\mathrm{e}^{-\phi(x)} \mathrm{e}^{\alpha^{\prime} i k_{1} \cdot X(x)} \lambda \otimes \sigma_{2}, \\
& V_{\phi}^{(-1)}(x)=\mathrm{e}^{-\phi(x)} \xi_{1 i} \psi^{i}(x) \mathrm{e}^{\alpha^{\prime} i q \cdot X(x)} \lambda \otimes \sigma_{3}, \\
& V_{\phi}^{(0)}(x)=\xi_{1 i}\left(\partial^{i} X(x)+i \alpha^{\prime} q \cdot \psi \psi^{i}(x)\right) \mathrm{e}^{\alpha^{\prime} i q \cdot X(x)} \lambda \otimes I, \\
& V_{C}^{\left(-\frac{3}{2},-\frac{1}{2}\right)}(z, \bar{z})=\left(P_{-} \mathscr{C}_{(n-1)} M_{p}\right)^{\alpha \beta} \mathrm{e}^{-3 \phi(z) / 2} \\
& \quad \times S_{\alpha}(z) \mathrm{e}^{i \frac{\alpha^{\prime}}{2} p \cdot X(z)} \mathrm{e}^{-\phi(\bar{z}) / 2} S_{\beta}(\bar{z}) \mathrm{e}^{i \frac{\alpha^{\prime}}{2} p \cdot D \cdot X(\bar{z})} \otimes \sigma_{1}, \\
& V_{C}^{\left(-\frac{1}{2},-\frac{1}{2}\right)}(z, \bar{z})=\left(P_{-} H_{(n)} M_{p}\right)^{\alpha \beta} \mathrm{e}^{-\phi(z) / 2} \\
& \quad \times S_{\alpha}(z) \mathrm{e}^{i \frac{\alpha^{\prime}}{2} p \cdot X(z)} \mathrm{e}^{-\phi(\bar{z}) / 2} S_{\beta}(\bar{z}) \mathrm{e}^{i \frac{\alpha^{\prime}}{2} p \cdot D \cdot X(\bar{z})} \otimes \sigma_{3} \sigma_{1} .
\end{aligned}
$$

$\sigma_{i}$ is a Pauli matrix. $\lambda$ is the external Chan-Paton matrix for the $\mathrm{U}(\mathrm{N})$ gauge group. The vertex operators of non-BPS Dbranes should accompany the internal degrees of freedom, given the fact that, if we send the tachyon to zero, one should recover the WZ action of the BPS branes. For more information we recommend Sect. 2 of [64].
This four point function at disk level can be computed if one takes into account the following on-shell conditions:

$q^{2}=p^{2}=0, \quad k_{1}^{2}=1 / 4, q \cdot \xi_{1}=0$.

The projection operator and the closed string RR field strength are defined by

$P_{-}=\frac{1}{2}\left(1-\gamma^{11}\right), \quad H_{(n)}=\frac{a_{n}}{n !} H_{\mu_{1} \ldots \mu_{n}} \gamma^{\mu_{1}} \ldots \gamma^{\mu_{n}}$.

The spinor notation is given by $\left(P_{-} H_{(n)}\right)^{\alpha \beta}=$ $C^{\alpha \delta}\left(P_{-} H_{(n)}\right)_{\delta}{ }^{\beta}$ where $\mathrm{C}$ is the charge conjugation matrix and for IIA (IIB) we pick up $n=2,4, a_{n}=i(n=1,3,5$, $a_{n}=1$ ), accordingly. If we employ the doubling trick, then one is able to just work with the holomorphic parts of the fields. Thus we apply the the following change of variables to our field content:

$$
\begin{aligned}
& \tilde{X}^{\mu}(\bar{z}) \rightarrow D_{v}^{\mu} X^{\nu}(\bar{z}), \\
& \tilde{\psi}^{\mu}(\bar{z}) \rightarrow D_{\nu}^{\mu} \psi^{\nu}(\bar{z}), \\
& \tilde{\phi}(\bar{z}) \rightarrow \phi(\bar{z}), \quad \text { and } \quad \tilde{S}_{\alpha}(\bar{z}) \rightarrow M_{\alpha}{ }^{\beta} S_{\beta}(\bar{z}),
\end{aligned}
$$

with

$D=\left(\begin{array}{cc}-1_{9-p} & 0 \\ 0 & 1_{p+1}\end{array}\right)$, and $M_{p}= \begin{cases}\frac{ \pm i}{(p+1) !} \gamma^{i_{1}} \gamma^{i_{2}} \ldots \gamma^{i_{p+1}} \epsilon_{i_{1} \ldots i_{p+1}} & \text { for } p \text { even } \\ \frac{ \pm 1}{(p+1) !} \gamma^{i_{1}} \gamma^{i_{2}} \ldots \gamma^{i_{p+1}} \gamma_{11} \epsilon_{i_{1} \ldots i_{p+1}} & \text { for } p \text { odd }\end{cases}$

Having carried the trick out, we would use the following propagators for all $X^{\mu}, \psi^{\mu}, \phi$ fields:

$$
\begin{aligned}
& \left\langle X^{\mu}(z) X^{\nu}(w)\right\rangle=-\frac{\alpha^{\prime}}{2} \eta^{\mu \nu} \log (z-w), \\
& \left\langle\psi^{\mu}(z) \psi^{\nu}(w)\right\rangle=-\frac{\alpha^{\prime}}{2} \eta^{\mu \nu}(z-w)^{-1}, \\
& \langle\phi(z) \phi(w)\rangle=-\log (z-w) .
\end{aligned}
$$

Hence, our amplitude in the asymmetric picture of RR is found to be

$$
\begin{aligned}
& \mathcal{A}^{C^{-2} T^{0} \phi^{0}}=\int \mathrm{d} x_{1} \mathrm{~d} x_{2} \mathrm{~d} x_{4} \mathrm{~d} x_{5} \\
& \quad \times\left(P_{-} \mathbb{C}_{(n-1)} M_{p}\right)^{\alpha \beta}\left(2 i \alpha^{\prime} k_{1 a} \xi_{2 i}\right)\left(x_{45}\right)^{-3 / 4}\left(I_{1}+I_{2}\right) \\
& \quad \times\left|x_{12}\right|^{\alpha^{\prime 2} k_{1} \cdot k_{2}}\left|x_{14} x_{15}\right|^{\frac{\alpha^{\prime 2}}{2} k_{1} \cdot p}\left|x_{24} x_{25}\right|^{\frac{\alpha^{\prime 2}}{2} k_{2} \cdot p}\left|x_{45}\right|^{\frac{\alpha^{\prime 2}}{4} p . D . p}
\end{aligned}
$$

with $x_{4}=z=x+i y, x_{5}=\bar{z}$ and

$$
I_{1}=-i p^{i}\left(\frac{x_{45}}{x_{24} x_{25}}\right) 2^{-1 / 2}\left(x_{14} x_{15}\right)^{-1 / 2}\left(x_{45}\right)^{-3 / 4}\left(\gamma^{a} C^{-1}\right)_{\alpha \beta} \text {. }
$$

To obtain the other correlation function including two spinors, a current and a fermion field $\left(I_{2}=2 i k_{2 b}<\right.$ : $\left.S_{\alpha}\left(x_{4}\right): S_{\beta}\left(x_{5}\right): \psi^{a}\left(x_{1}\right): \psi^{b} \psi^{i}\left(x_{2}\right):>\right)$ we use the so-called Wick-like formula [65] to get 


$$
\begin{aligned}
I_{2}= & \left(\left(\Gamma^{i b a} C^{-1}\right)_{\alpha \beta}-2 \eta^{a b}\left(\gamma^{i} C^{-1}\right)_{\alpha \beta} \frac{2 \operatorname{Re}\left[x_{14} x_{25}\right]}{x_{12} x_{45}}\right) \\
& \times 2 i k_{2 b} 2^{-3 / 2}\left(x_{24} x_{25}\right)^{-1}\left(x_{14} x_{15}\right)^{-1 / 2}\left(x_{45}\right)^{1 / 4} .
\end{aligned}
$$

One could precisely show that now the amplitude is $S L(2, R)$ invariant and to remove the volume of conformal Killing group we do gauge fixing as $\left(x_{1}, x_{2}, z, \bar{z}\right)=(x,-x, i,-i)$ with the Jacobian $J=-2 i\left(1+x^{2}\right)$. Setting the above gauge fixing, we see that the second term of $I_{2}$ does not have any contribution to the final result of the amplitude due to the fact that the integrand is odd, while the moduli space is covered on the entire space-time or due to having a symmetric interval. $u=-\frac{\alpha^{\prime}}{2}\left(k_{1}+k_{2}\right)^{2}$ is introduced and the amplitude results,

$$
\begin{aligned}
\mathcal{A}^{C^{-2} T^{0} \phi^{0}}= & \int_{-\infty}^{\infty} \mathrm{d} x(2 x)^{-2 u-1 / 2}\left(1+x^{2}\right)^{-1 / 2+2 u} \\
& \times\left(p^{i} \operatorname{Tr}\left(P_{-} \mathbb{C}_{(n-1)} M_{p} \gamma^{a}\right)\right. \\
& \left.+i k_{2 b} \operatorname{Tr}\left(P_{-} \mathbb{C}_{(n-1)} M_{p} \Gamma^{i b a}\right)\right) k_{1 a} \xi_{2 i} .
\end{aligned}
$$

The ultimate result of the amplitude is given by

$$
\begin{aligned}
\mathcal{A}^{C^{-2} T^{0} \phi^{0}}=( & p^{i} \operatorname{Tr}\left(P_{-} \mathscr{C}_{(n-1)} M_{p} \gamma^{a}\right) \\
& \left.+i k_{2 b} \operatorname{Tr}\left(P_{-} \mathbb{C}_{(n-1)} M_{p} \Gamma^{i b a}\right)\right) k_{1 a} \xi_{2 i} \\
& \times\left(\pi \beta^{\prime} \mu_{p}^{\prime}\right) 2 \sqrt{\pi} \frac{\Gamma[-u+1 / 4]}{\Gamma[3 / 4-u]} .
\end{aligned}
$$

$\mu_{p}^{\prime}$ We have the RR charge of the brane. All the traces are nonzero for the $p+1=n$ case and can be calculated as

$$
\begin{aligned}
& \operatorname{Tr}\left(\mathscr{C}_{(n-1)} M_{p}\left(k_{1} \cdot \gamma\right)\right)= \pm \frac{32}{p !} \epsilon^{a_{0} \ldots a_{p-1} a} C_{a_{0} \ldots a_{p-1}} k_{1 a} \\
& \operatorname{Tr}\left(\mathscr{C}_{(n-1)} M_{p}(\xi \cdot \gamma)\left(k_{2} \cdot \gamma\right)\left(k_{1} \cdot \gamma\right)\right) \\
& = \pm \frac{32}{p !} \epsilon^{a_{0} \ldots a_{p-2} b a} C_{a_{0} \ldots a_{p-2}} k_{1 a} k_{2 b} \xi_{1 i} .
\end{aligned}
$$

The correct expansion of the amplitude can be found by dealing with either massless or tachyon poles of the amplitude. From a three point function including an RR and a real tachyon and using its momentum conservation along the world volume of the brane, $k^{2}=p^{a} p_{a}=\frac{1}{4}$ [66], one realizes that this constraint holds for the $C T \phi$ amplitude and indeed the proper momentum expansion can be read off as follows:

$u=-p^{a} p_{a} \rightarrow \frac{-1}{4}$,

$\sqrt{\pi} \frac{\Gamma[-u+1 / 4]}{\Gamma[3 / 4-u]}=\pi \sum_{n=-1}^{\infty} c_{n}(u+1 / 4)^{n+1}$,

where the first three coefficients are

$c_{-1}=1, c_{0}=2 \ln (2), c_{1}=\frac{1}{6}\left(\pi^{2}+12 \ln (2)^{2}\right)$.
The first term in (8) can be produced by using the following Chern-Simons coupling, where the scalar field has been taken from the Taylor expansion:

$S_{1}=\frac{2 i \beta^{\prime} \mu_{p}^{\prime}}{p !}\left(2 \pi \alpha^{\prime}\right)^{2} \int_{\Sigma_{p+1}} \partial_{i} C_{p} \wedge \mathrm{DT} \phi^{i}$.

Note that the second term of (8) can just be produced if one introduces a new coupling where this time a scalar field comes from the pull-back of the brane and the covariant derivative of the tachyon appears to cover the entire $(p+1)$ world volume direction. Hence the second term of (8) can be regenerated by the following new mixed WZ and pull-back coupling:

$$
S_{2}=\frac{2 i \beta^{\prime} \mu_{p}^{\prime}}{(p) !}\left(2 \pi \alpha^{\prime}\right)^{2} \int_{\Sigma_{p+1}} \epsilon^{a_{0} . a_{p}} C_{i a_{0} \ldots a_{p-2}} D_{a_{p-1}} T D_{a_{p}} \phi^{i} .
$$

As we have seen, the expansion of the amplitude has an infinite contact interaction and all those contact interaction terms related to the first term of (8) can be produced in the EFT by applying all infinite higher derivative corrections to the WZ effective actions of a real tachyon, a scalar field and a $C_{p} \mathrm{RR}$ closed string, see (9),

$$
\begin{aligned}
& \frac{2 i \beta^{\prime} \mu_{p}^{\prime}}{p !}\left(2 \pi \alpha^{\prime}\right)^{2} \int_{\Sigma_{p+1}} \partial_{i} C_{p} \wedge \operatorname{Tr} \\
& \times\left(\sum_{n=-1}^{\infty} c_{n}\left(\alpha^{\prime}\right)^{n+1} D_{a_{1}} \ldots D_{a_{n+1}} \mathrm{DT} D^{a_{1}} \ldots D^{a_{n+1}} \phi^{i}\right) .
\end{aligned}
$$

Likewise all the contact interactions related to the second term (8) can be constructed if one applies the same prescription to all higher derivative corrections to $S_{2}$ action as follows:

$$
\begin{aligned}
& \frac{2 i \beta^{\prime} \mu_{p}^{\prime}}{p !}\left(2 \pi \alpha^{\prime}\right)^{2} \int_{\Sigma_{p+1}} \epsilon^{a_{0} \ldots a_{p}} C_{i a_{0} \ldots a_{p-2}} \operatorname{Tr} \\
& \times\left(\sum_{n=-1}^{\infty} c_{n}\left(\alpha^{\prime}\right)^{n+1} D_{a_{1}} \ldots D_{a_{n+1}} D_{a_{p-1}} T D^{a_{1}} \ldots D^{a_{n+1}} D_{a_{p}} \phi^{i}\right) .
\end{aligned}
$$

It is also interesting to revisit the amplitude in the other pictures. The final result of the amplitude can be derived:

$$
\begin{aligned}
\mathcal{A}^{C^{-1} T^{0} \phi^{-1}=} & 2 \operatorname{Tr}\left(P_{-} H_{(n)} M_{p} \Gamma^{i a}\right) k_{1 a} \xi_{2 i}\left(\pi \beta^{\prime} \mu_{p}^{\prime}\right) \\
& \times \sqrt{\pi} \frac{\Gamma[-u+1 / 4]}{\Gamma[3 / 4-u]} .
\end{aligned}
$$

The trace that includes the $\gamma^{11}$ factor has the special property that all results hold for the following relations as well:

$p>3, H_{n}=* H_{10-n}, n \geq 5$.

Now if we apply momentum conservation $\left(k_{1}+k_{2}+p\right)^{a}=0$ to the above amplitude then we realize that the amplitude (12) can just produce the first term of (8); more importantly one 
finds that a Bianchi identity holds for the world volume of the branes in the presence of the RR field strength,

$p_{a} H_{a_{0} \ldots a_{p-1}} \epsilon^{a_{0} \ldots a_{p-1} a}=0$.

Finally, the result of the amplitude for $\mathcal{A}^{T^{-1} \phi^{0} C^{-1}}$ is derived to be

$$
\begin{aligned}
& \left(k_{2 b} \operatorname{Tr}\left(P_{-} H_{(n)} M_{p} \Gamma^{i b}\right)-p^{i} \operatorname{Tr}\left(P_{-} H_{(n)} M_{p}\right)\right) \\
& \quad \times \xi_{2 i}\left(2 \pi \beta^{\prime} \mu_{p}^{\prime}\right) \sqrt{\pi} \frac{\Gamma[-u+1 / 4]}{\Gamma[3 / 4-u]} .
\end{aligned}
$$

Finding the above result and keeping in mind momentum conservation, one understands that, to get a result consistent with both the string theory and the effective field theory parts, the restricted world volume Bianchi identity (13) has to be modified, to obtain a new Bianchi identity which will be valid for both world volume and transverse directions of the branes as follows:

$p^{i} \epsilon^{a_{0} \ldots a_{p}} H_{a_{0} \ldots a_{p}}+p^{a} \epsilon^{a_{0} \ldots a_{p-1} a} H_{a_{0} \ldots a_{p-1}}^{i}=0$.

\section{$3<V_{C^{-1}} V_{\phi^{-1}} V_{T^{0}} V_{T^{0}} V_{T^{0}}>$ amplitude}

In this section we would like to deal with a non-BPS six point function including an RR, a transverse scalar field and three real tachyons to be able to find not only the proper expansion of the amplitude but also to reveal all the bulk singularity structures as well as various restricted Bianchi identities. Given the exact symmetries of the string theory amplitudes, a tachyon expansion and the particular soft limit, in the following we show that one is able to predict some of the singularity structures of $<$ $V_{C^{-1}(z, \bar{z})} V_{\phi^{-1}\left(x_{1}\right)} V_{T^{0}\left(x_{2}\right)} V_{T^{0}\left(x_{3}\right)} V_{T^{0}\left(x_{4}\right)}>$ amplitude. We then work out $<V_{C^{-1}(z, \bar{z})} V_{\phi^{0}\left(x_{1}\right)} V_{T^{-1}\left(x_{2}\right)} V_{T^{0}\left(x_{3}\right)} V_{T^{0}\left(x_{4}\right)}>$ and determine all the singularities including the bulk singularities that carry the momentum of RR in the bulk directions. One needs to provide the correlation function between two spinors and four fermion fields at different locations where just one of them moves along a transverse direction of the brane so $I_{1}^{c b a i}=<: S_{\alpha}\left(x_{z}\right): S_{\beta}\left(x_{\bar{z}}\right): \psi^{i}\left(x_{1}\right): \psi^{a}\left(x_{2}\right)$ : $\psi^{b}\left(x_{3}\right): \psi^{c}\left(x_{4}\right):>$ is found to be

$$
\begin{aligned}
I_{1}^{c b a i}= & \left\{\left(\Gamma^{c b a i} C^{-1}\right)_{\alpha \beta}-\alpha^{\prime} \eta^{a b}\left(\Gamma^{c i} C^{-1}\right)_{\alpha \beta} \frac{\operatorname{Re}\left[x_{25} x_{36}\right]}{x_{23} x_{56}}\right. \\
& +\alpha^{\prime} \eta^{a c}\left(\Gamma^{b i} C^{-1}\right)_{\alpha \beta} \frac{\operatorname{Re}\left[x_{25} x_{46}\right]}{x_{24} x_{56}} \\
& \left.\left.-\alpha^{\prime} \eta^{b c}\left(\Gamma^{a i} C^{-1}\right)_{\alpha \beta} \frac{\operatorname{Re}\left[x_{35} x_{46}\right]}{x_{34} x_{56}}\right)\right\} 2^{-2} x_{45}^{3 / 4} \\
& \times\left(x_{15} x_{16} x_{25} x_{26} x_{35} x_{36} x_{45} x_{46}\right)^{-1 / 2} .
\end{aligned}
$$

Note that here $x_{5}=z=x+i y, x_{6}=\bar{z}$. All the techniques have already been explained, fixing the position of open strings at $x_{1}=0,0 \leq x_{2} \leq 1, x_{3}=1, x_{4}=\infty$ and using six independent Mandelstam variables, $s=-\left(\frac{1}{4}+2 k_{1} \cdot k_{3}\right), t=$ $-\left(\frac{1}{4}+2 k_{1} \cdot k_{2}\right), v=-\left(\frac{1}{4}+2 k_{1} \cdot k_{4}\right), u=-\left(\frac{1}{2}+2 k_{2} \cdot k_{3}\right), r=$ $-\left(\frac{1}{2}+2 k_{2} \cdot k_{4}\right), w=-\left(\frac{1}{2}+2 k_{3} \cdot k_{4}\right)$. The final form of the amplitude is written

$$
\begin{aligned}
\mathcal{A} & =4 i \xi_{1 i}\left(P_{-} H_{(n)} M_{p}\right)^{\alpha \beta} \int_{0}^{1} \mathrm{~d} x_{2} x_{2}^{-2 t-1 / 2}\left(1-x_{2}\right)^{-2 u-1} \\
& \times \int d z \int d \bar{z}|1-z|^{2 s+2 u+2 w+1 / 2}|z|^{2 t+2 s+2 v-1 / 2} \\
& \times k_{2 a} k_{3 b} k_{4 c}(z-\bar{z})^{-2(t+s+u+v+r+w)-5 / 2} \\
& \times\left|x_{2}-z\right|^{2 t+2 u+2 r+1 / 2} \\
& \times\left[\left(\Gamma^{c b a i} C^{-1}\right)_{\alpha \beta}+(z-\bar{z})^{-1}\right. \\
& \times\left(2 \eta^{a b}\left(\Gamma^{c i} C^{-1}\right)_{\alpha \beta}\left(1-x_{2}\right)^{-1}\left(x_{2}-x x_{2}-x+|z|^{2}\right)\right. \\
& \left.\left.-2 \eta^{a c}\left(\Gamma^{b i} C^{-1}\right)_{\alpha \beta}\left(x_{2}-x\right)+2 \eta^{b c}\left(\Gamma^{a i} C^{-1}\right)_{\alpha \beta}(1-x)\right)\right] .
\end{aligned}
$$

The amplitude makes sense for the $p=n+1$ and $p+1=$ $n$ cases. The algebraic form of the above integrals can be derived in the soft limit, $4 k_{2} \cdot p \rightarrow 1$. Using this limit and Appendix B of [66] and [67] one arrives at a closed form for the integrals. For simplicity, we just write down the ultimate result of the amplitude for $p=n+1$ case as

$$
\mathcal{A}_{1}^{C \phi T T T}=4 i \xi_{1 i} \pi k_{2 a} k_{3 b} k_{4 c} \operatorname{Tr}\left(P_{-} \mathbb{C}_{(n-1)} M_{p} \Gamma^{c b a i}\right) M_{1} M_{2}
$$

where $M_{1}, M_{2}$ are

$$
\begin{aligned}
& M_{1}=(2)^{-2(t+s+u+v+r+w)-5 / 2} \frac{\Gamma\left(-2 t+\frac{1}{2}\right) \Gamma(-2 u)}{\Gamma\left(-2 t-2 u+\frac{1}{2}\right)} \\
& M_{2}=\frac{\Gamma\left(-u-r-w-\frac{1}{2}\right) \Gamma(-t-v-r) \Gamma\left(-s+r+\frac{1}{4}\right) \Gamma\left(-t-s-u-v-r-w-\frac{3}{4}\right)}{\Gamma\left(-u-s-w-\frac{1}{4}\right) \Gamma\left(-t-s-v+\frac{1}{4}\right) \Gamma\left(-u-w-t-v-2 r-\frac{1}{2}\right)} .
\end{aligned}
$$


The other part of the amplitude holds for the $C_{p}$ case and one reveals its final form as follows:

$$
\begin{aligned}
\mathcal{A}_{2}= & M_{1} \pi \frac{32}{(p+1) !} \epsilon^{a_{0} \ldots a_{p-1} a} H_{a_{0} \ldots a_{p-1}}^{i} \xi_{1 i} i \\
& \left\{-k_{2 a}\left(w+\frac{1}{2}\right)\left(-r-t-v-\frac{1}{2}\right) M_{3}\right. \\
& +\frac{1}{4\left(-2 t-2 u+\frac{1}{2}\right)} k_{3 a}\left(r+\frac{1}{2}\right) M_{3} \\
& \times((-1+r(-2+8 t-8 u)-2 v+2 t(1+4 t+4 v) \\
& -8 u(1+u+w))) \\
& +\frac{1}{16} k_{4 a} M_{4}(4 s(-1+4 t)+4(5+4 r) u+8 r+3 \\
& +20 t+4 w+16(t+u)(u+w))\}
\end{aligned}
$$

where $M_{3}, M_{4}$ are written in terms of the ratio of the Gamma functions, where

$$
\begin{aligned}
& l_{1}=2 \eta^{a b}\left(\Gamma^{c i} C^{-1}\right)_{\alpha \beta}-2 \eta^{a c}\left(\Gamma^{b i} C^{-1}\right)_{\alpha \beta}-2 \eta^{b c}\left(\Gamma^{i a} C^{-1}\right)_{\alpha \beta} \\
& l_{2}=-2 \eta^{a b}\left(\Gamma^{c i} C^{-1}\right)_{\alpha \beta} \\
& l_{3}=2 \eta^{b c}\left(\Gamma^{i a} C^{-1}\right)_{\alpha \beta} .
\end{aligned}
$$

The amplitude makes sense for the $p=n+1$ and $p+$ $1=n$ cases. Using the soft limit, $4 k_{2} \cdot p \rightarrow 1$, one finds the amplitude for the $p=n+1$ case,

$$
\begin{aligned}
& \mathcal{A}_{1}^{C^{-1} \phi^{0} T^{-1} T^{0} T^{0}} \sim \frac{64 i \xi_{1 i} \pi M_{1} M_{2} k_{3 b} k_{4 c}}{(p-1) !} \\
&\left(k_{1 a} \epsilon^{a_{0} \ldots a_{p-3} a c b} H_{a_{0} \ldots a_{p-3}}^{i}+p^{i} \epsilon^{a_{0} \ldots a_{p-2} c b} H_{a_{0} \ldots a_{p-2}}\right) .
\end{aligned}
$$

The second part of the amplitude holds for the $C_{p}$ case and one finds

$$
\begin{aligned}
M_{3} & =\frac{\Gamma\left(r-s+\frac{3}{4}\right) \Gamma\left(-t-v-r-\frac{1}{2}\right) \Gamma(-u-r-w-1) \Gamma\left(-t-s-u-v-r-w-\frac{5}{4}\right)}{\Gamma\left(-t-s-v+\frac{1}{4}\right) \Gamma\left(-u-s-w-\frac{1}{4}\right) \Gamma\left(-t-u-v-w-2 r-\frac{1}{2}\right)} \\
M_{4} & =\frac{\Gamma\left(r-s-\frac{1}{4}\right) \Gamma\left(-t-v-r+\frac{1}{2}\right) \Gamma(-u-r-w-1) \Gamma\left(-t-s-u-v-r-w-\frac{5}{4}\right)}{\Gamma\left(-t-s-v+\frac{1}{4}\right) \Gamma\left(-u-s-w-\frac{1}{4}\right) \Gamma\left(-t-u-v-w-2 r-\frac{1}{2}\right)} .
\end{aligned}
$$

Let us deal with the bulk singularities.

$$
\begin{aligned}
\mathcal{A}_{2}= & M_{1} \pi \frac{32}{(p) !} \xi_{1 i} i \\
& \left\{-p^{i} \epsilon^{a_{0} \ldots a_{p}} H_{a_{0} \ldots a_{p}}\left(w+\frac{1}{2}\right)\left(-r-t-v-\frac{1}{2}\right) M_{3}\right. \\
& +\epsilon^{a_{0} \ldots a_{p-1} d} H_{a_{0} \ldots a_{p-1}}^{i} M_{4} \\
& \left(k_{4 d}\left(s+\frac{1}{4}\right)(-u-r-w-1)\right. \\
& -k_{1 d}\left(w+\frac{1}{2}\right)\left(r-s-\frac{1}{4}\right) \\
& \left.\left.+k_{3 d} \frac{\left(r-s-\frac{1}{4}\right)}{\left(-r-t-v-\frac{1}{2}\right)}\left(v+\frac{1}{4}\right)(-u-r-w-1)\right)\right\} .
\end{aligned}
$$

$$
\begin{aligned}
\mathcal{A} & \sim 4 i \xi_{1 i}\left(P_{-} H_{(n)} M_{p}\right)^{\alpha \beta} \int_{0}^{1} \mathrm{~d} x_{2} x_{2}^{-2 t-1 / 2}\left(1-x_{2}\right)^{-2 u-1} \\
& \times \int d z \int d \bar{z}|1-z|^{2 s+2 u+2 w+1 / 2}|z|^{2 t+2 s+2 v-1 / 2} \\
& \times k_{3 b} k_{4 c}(z-\bar{z})^{-2(t+s+u+v+r+w)-5 / 2}\left|x_{2}-z\right|^{2 t+2 u+2 r+1 / 2} \\
& \times\left[p^{i}\left(2 \eta^{b c}\left(C^{-1}\right)_{\alpha \beta} \frac{1-x}{(z-\bar{z})} v+\left(\Gamma^{c b} C^{-1}\right)_{\alpha \beta}\right)\right. \\
& +k_{1 a}\left(\left(\Gamma^{c b i a} C^{-1}\right)_{\alpha \beta}+(z-\bar{z})^{-1}\right. \\
& \left.\left.\times\left(x l_{1}+l_{2}|z|^{2}+l_{3}\right)\right)\right]
\end{aligned}
$$

Now let us deal with all singularities and start to produce them.

\section{Bulk Singularity Structures}

To obtain all the singularity structures, including the ones that carry momentum of the closed string RR in the bulk direction, one needs to find first the expansion of the amplitude. The following remarks need to be taken into con- 
sideration. Having applied momentum conservation we get $s+t+u+v+r+w=-p^{a} p_{a}-\frac{3}{2}$. Using the constraint $p^{a} p^{a} \rightarrow \frac{1}{4}$ for the non-BPS branes, taking the symmetries of our amplitude (given the EFT and the fact that it should be symmetric under exchanging $s, t, v$ ), one obtains the expansion for the amplitude (20) as follows:

$$
\left(s, t, v \rightarrow-\frac{1}{4}\right),(u, r \rightarrow 0), w \rightarrow-1 .
$$

The expansion of $\left(M_{1} M_{2}\right)$ for this particular soft limit and around (21) can be read off:

$$
\begin{aligned}
& -\frac{\pi^{3 / 2}}{u}-\frac{\pi^{7 / 2}}{6 u}\left((t+s+r+w)^{2}+2 t(v-w)\right. \\
& \left.-2 s(t+w)+2 r(r+v)+v^{2}+\ldots\right) .
\end{aligned}
$$

Given the above expansion, and standard EFT methods that propose infinite u-channel massless gauge field poles and symmetries [68], one understands that $k_{i} . k_{j} \rightarrow 0$ for massless strings and that $p^{a} p^{a} \rightarrow \frac{1}{4}$ for non-BPS branes. There is no coupling between two tachyons and a scalar field. Given the selection rules for non-BPS branes [54] and the fact that the kinetic term of tachyon is fixed in the DBI action, one clarifies that there is no double pole for the $p=n+1$ case. This point can also be observed from the expansion of the amplitude; hence we have an infinite number of $\mathrm{u}$ channel simple poles. For the $p=n+1$ case, from EFT and the above expansion, one notices that the S-matrix has an infinite number of $\mathrm{u}$-channel gauge field poles. The first $\mathrm{u}$-channel pole in string theory can be written down:

$\frac{64 i \pi^{3 / 2}}{(p-1) ! u} \epsilon^{a_{0} \ldots a_{p-3} a c b} H_{a_{0} \ldots a_{p-3}}^{i} k_{1 a} k_{3 b} k_{4 c} \xi_{1 i} \operatorname{Tr}\left(\lambda_{1} \lambda_{2} \lambda_{3} \lambda_{4}\right)$.

This simple u-channel pole can be reconstructed in an EFT by the following sub amplitude:

$$
\begin{aligned}
& V_{a}^{\alpha}\left(T_{2}, T_{3}, A\right) G_{a b}^{\alpha \beta}(A) V_{b}^{\beta}\left(C_{p-2}, A, \phi_{1}, T_{4}\right) \\
& V_{a}^{\alpha}\left(T_{2}, T_{3}, A\right)=i T_{p}\left(2 \pi \alpha^{\prime}\right)\left(k_{2}-k_{3}\right)_{a} \operatorname{Tr}\left(\lambda_{2} \lambda_{3} \lambda^{\alpha}\right) \\
& G^{a b}(A)=\frac{i \delta^{a b} \delta^{\alpha \beta}}{\left(2 \pi \alpha^{\prime}\right)^{2} T_{p} u} \\
& V_{b}^{\beta}\left(C_{p-2}, A, \phi_{1}, T_{4}\right)=i \mu_{p}^{\prime} \beta^{\prime}\left(2 \pi \alpha^{\prime}\right)^{3} \\
& \frac{1}{(p-1) !} \epsilon^{a_{0} \ldots a_{p-1} b} H_{a_{0} \ldots a_{p-3}}^{i} k_{4 a_{p-2}} k_{1 a_{p-1}} \xi_{1 i} \operatorname{Tr}\left(\lambda_{4} \lambda_{1} \lambda^{\beta}\right) .
\end{aligned}
$$

Here $\alpha, \beta$ are gauge group indices. Now if we use the above vertices in an EFT and make use of momentum conservation, $\left(k_{1}+k_{2}+k_{3}+k_{4}+p\right)^{a}=0$, the Bianchi identity, $p_{a_{p-2}} H_{a_{0} \ldots a_{p-3}}=0$, the fact that the amplitude is symmetric under $k_{1 a_{p-1}} k_{1 a_{p-2}}$, and also due to the antisymmetry property of $\epsilon$, we realize that the term $k_{1 a_{p-1}} k_{1 a_{p-2}}$ does not have any effect on the EFT part of the amplitude. If we multiply (23) by $\frac{1}{2} \mu_{p}^{\prime} \beta^{\prime} \pi^{1 / 2}$ and compare it with the above EFT amplitude, we then can explore the fact that the first simple $\mathrm{u}$-channel gauge field pole is exactly generated.

$V^{a}\left(C_{p-2}, A, \phi_{1}, T_{4}\right)$ was derived from some part of the mixed WZ coupling and Taylor expansion,

$\beta^{\prime} \mu_{p}^{\prime}\left(2 \pi \alpha^{\prime}\right)^{3} \int_{\Sigma_{p+1}} \operatorname{Tr}\left(\partial_{i} C_{p-2} \wedge F \wedge \mathrm{DT} \phi^{i}\right)$.

However, as can be seen from the expansion, the amplitude in (19) has an infinite number of bulk singularity structures, concretely, where the first bulk pole in string theory reads

$\frac{32 i \beta^{\prime} \mu_{p}^{\prime} \pi^{2} \xi_{1 i} k_{3 b} k_{4 c}}{(p-1) ! u} p^{i} \epsilon^{a_{0} \ldots a_{p-2} c b} H_{a_{0} \ldots a_{p-2}}$.

If one wants to extract the couplings from (25), one performs integration by parts to arrive at two different contributions:

$$
\begin{aligned}
& -\beta^{\prime} \mu_{p}^{\prime}\left(2 \pi \alpha^{\prime}\right)^{3} \int_{\Sigma_{p+1}} \epsilon^{a_{0} \ldots a_{p}}, \\
& \quad \times\left(d_{a_{p-2}} \partial_{i} C_{a_{0} \ldots a_{p-3}} A_{a_{p-1}} d_{a_{p}} T \phi^{i}-\partial_{i} C_{a_{0} \ldots a_{p-3}} A_{a_{p-1}} d_{a_{p}} T d_{a_{p-2}} \phi^{i}\right),
\end{aligned}
$$

where to derive the first simple u-channel gauge pole given in (23), we have already used the contribution from the second term of (27). Now if we use the relation

$(p-2) \partial_{i} C_{a_{0} \ldots a_{p-3}}=H_{a_{0} \ldots a_{p-3}}^{i}-\partial_{\left[a_{p-3}\right.} C_{\left.a_{0} \ldots a_{p-4}\right] i}$

and plug it into the first term of (27), then we would be able to produce the bulk part of the vertex $V^{b}\left(C_{p-2}, A, \phi_{1}, T_{4}\right)$. Eventually by replacing it into the same EFT sub amplitude (24) we are able to precisely produce the first bulk singularity u-channel pole (26) which carries the $p$. $\xi$ term as well. As one notices from the expansion of the amplitude, we have an infinite number of u-channel poles and to generate them in an EFT the following remarks are in order.

The vertex of $V_{a}^{\alpha}\left(T_{2}, T_{3}, A\right)$ comes from the kinetic term of tachyons in the DBI action which is fixed and has no correction. The propagator is also fixed, as it comes from the kinetic term of gauge fields that has been fixed for this case too. Therefore, to be able to reconstruct all infinite $\mathrm{u}$-channel poles one must directly apply infinite higher derivative corrections to the mixed $\mathrm{WZ}$ coupling (25), as follows:

$$
\begin{aligned}
& \beta^{\prime} \mu_{p}^{\prime}\left(2 \pi \alpha^{\prime}\right)^{3} \sum_{n=-1}^{\infty} b_{n} \\
& \times \int_{\Sigma_{p+1}} \operatorname{Tr}\left(\partial_{i} C_{p-2} \wedge D^{b_{1}} \ldots D^{b_{n}} F \wedge D_{b_{1}} \ldots D_{b_{n}}\left[\mathrm{DT} \phi^{i}\right]\right) .
\end{aligned}
$$


Keeping fixed $V_{a}^{\alpha}\left(T_{2}, T_{3}, A\right)$ and the propagator, extracting the modified all order vertex $V^{b}\left(C_{p-2}, A, \phi_{1}, T_{4}\right)$ from (29) and replacing it in the EFT amplitude (24), then one is able to show that all infinite bulk singularity structures are precisely produced. This clearly confirms that the expansion is consistent with the EFT amplitude as well.

The amplitude has an infinite number of massless scalar poles in the $\left(t^{\prime}+v^{\prime}+r\right)^{2}$ channel that correspond to the extensions of higher derivative corrections of two tachyontwo scalar field couplings. These corrections originate from the second part of the amplitude in (20). They are reconstructed by the following EFT prescription:

$$
\begin{aligned}
& \mathcal{A}=V_{i}^{\alpha}\left(C_{p}, T_{3}, \phi\right) G_{i j}^{\alpha \beta}(\phi) V_{j}^{\beta}\left(\phi, T_{2}, \phi_{1}, T_{4}\right) \\
& G_{i j}^{\alpha \beta}(\phi)=\frac{i \delta_{\alpha \beta} \delta_{i j}}{\left(2 \pi \alpha^{\prime}\right)^{2} T_{p}\left(t^{\prime}+v^{\prime}+r\right)} \\
& V_{i}^{\alpha}\left(C_{p}, T_{3}, \phi\right)=\mu_{p}^{\prime} \beta^{\prime}\left(2 \pi \alpha^{\prime}\right)^{2} \\
& \quad \times \frac{1}{(p) !} \epsilon^{a_{0} \ldots a_{p}} H_{a_{0} \ldots a_{p-1}}^{i} k_{3 a_{p}} \operatorname{Tr}\left(\lambda_{3} \Lambda^{\alpha}\right) .
\end{aligned}
$$

To generate all scalar poles at first order one needs to employ the following Lagrangian:

$$
\begin{aligned}
& \mathcal{L}(\phi, \phi, T, T)=-2 T_{p}\left(\pi \alpha^{\prime}\right)^{3} \mathrm{STr} \\
& \quad\left(m^{2} T^{2}\left(D_{a} \phi^{i} D^{a} \phi_{i}\right)+\frac{\alpha^{\prime}}{2} D^{\alpha} T D_{\alpha} T D_{a} \phi^{i} D^{a} \phi_{i}\right. \\
& \left.-\alpha^{\prime} D^{b} T D^{a} T D_{a} \phi^{i} D_{b} \phi_{i}\right),
\end{aligned}
$$

while to produce all the other poles, one needs to know higher derivative corrections to the two tachyon-two scalar field couplings to all orders,

$$
\begin{aligned}
\mathcal{L}= & -2 T_{p}\left(\pi \alpha^{\prime}\right)\left(\alpha^{\prime}\right)^{2+n+m} \\
& \sum_{n, m=0}^{\infty}\left(\mathcal{L}_{1}^{n m}+\mathcal{L}_{2}^{n m}+\mathcal{L}_{3}^{n m}+\mathcal{L}_{4}^{n m}\right),
\end{aligned}
$$

where

$$
\begin{aligned}
\mathcal{L}_{1}^{n m}= & m^{2} \operatorname{Tr}\left(a _ { n , m } \left[\mathcal{D}_{n m}\left(T^{2} D_{a} \phi^{i} D^{a} \phi_{i}\right)\right.\right. \\
& \left.+\mathcal{D}_{n m}\left(D_{a} \phi^{i} D^{a} \phi_{i} T^{2}\right)\right] \\
& +b_{n, m}\left[\mathcal{D}_{n m}^{\prime}\left(T D_{a} \phi^{i} T D^{a} \phi_{i}\right)\right. \\
& \left.\left.+\mathcal{D}_{n m}^{\prime}\left(D_{a} \phi^{i} T D^{a} \phi_{i} T\right)\right]+ \text { h.c. }\right), \\
\mathcal{L}_{2}^{n m}= & \operatorname{Tr}\left(a _ { n , m } \left[\mathcal{D}_{n m}\left(D^{\alpha} T D_{\alpha} T D_{a} \phi^{i} D^{a} \phi_{i}\right)\right.\right. \\
& \left.+\mathcal{D}_{n m}\left(D_{a} \phi^{i} D^{a} \phi_{i} D^{\alpha} T D_{\alpha} T\right)\right] \\
& +b_{n, m}\left[\mathcal{D}_{n m}^{\prime}\left(D^{\alpha} T D_{a} \phi^{i} D_{\alpha} T D^{a} \phi_{i}\right)\right. \\
& \left.\left.+\mathcal{D}_{n m}^{\prime}\left(D_{a} \phi^{i} D_{\alpha} T D^{a} \phi_{i} D^{\alpha} T\right)\right]+ \text { h.c. }\right),
\end{aligned}
$$

$\overline{2 \text { We have } t^{\prime}=} t+\frac{1}{4}, v^{\prime}=v+\frac{1}{4}$

$$
\begin{aligned}
\mathcal{L}_{3}^{n m}= & -\operatorname{Tr}\left(a _ { n , m } \left[\mathcal{D}_{n m}\left(D^{\beta} T D_{\mu} T D^{\mu} \phi^{i} D_{\beta} \phi_{i}\right)\right.\right. \\
& \left.+\mathcal{D}_{n m}\left(D^{\mu} \phi^{i} D_{\beta} \phi_{i} D^{\beta} T D_{\mu} T\right)\right] \\
& +b_{n, m}\left[\mathcal{D}_{n m}^{\prime}\left(D^{\beta} T D^{\mu} \phi^{i} D_{\mu} T D_{\beta} \phi_{i}\right)\right. \\
& \left.\left.+\mathcal{D}_{n m}^{\prime}\left(D^{\mu} \phi^{i} D_{\mu} T D_{\beta} \phi_{i} D^{\beta} T\right)\right]+ \text { h.c. }\right), \\
\mathcal{L}_{4}^{n m}= & -\operatorname{Tr}\left(a _ { n , m } \left[\mathcal{D}_{n m}\left(D^{\beta} T D^{\mu} T D_{\beta} \phi^{i} D_{\mu} \phi_{i}\right)\right.\right. \\
& \left.+\mathcal{D}_{n m}\left(D^{\beta} \phi^{i} D^{\mu} \phi_{i} D_{\beta} T D_{\mu} T\right)\right] \\
& +b_{n, m}\left[\mathcal{D}_{n m}^{\prime}\left(D^{\beta} T D_{\beta} \phi^{i} D^{\mu} T D_{\mu} \phi_{i}\right)\right. \\
& \left.\left.+\mathcal{D}_{n m}^{\prime}\left(D_{\beta} \phi^{i} D_{\mu} T D^{\mu} \phi_{i} D^{\beta} T\right)\right]+ \text { h.c. }\right) .
\end{aligned}
$$

The definitions for the higher derivative operators $D_{n m}$ and $D_{n m}^{\prime}$ are

$$
\begin{aligned}
D_{n m}(\mathrm{EFGH}) \equiv & D_{b_{1}} \ldots D_{b_{m}} D_{a_{1}} \\
& \ldots D_{a_{n}} E F D^{a_{1}} \ldots D^{a_{n}} G D^{b_{1}} \ldots D^{b_{m}} H, \\
D_{n m}^{\prime}(\mathrm{EFGH}) \equiv & D_{b_{1}} \ldots D_{b_{m}} D_{a_{1}} \\
& \ldots D_{a_{n}} E D^{a_{1}} \ldots D^{a_{n}} F G D^{b_{1}} \ldots D^{b_{m}} H .
\end{aligned}
$$

The all order extended vertex $V_{j}^{\beta}\left(\phi, T_{2}, \phi_{1}, T_{4}\right)$ is derived from (33) and in momentum space takes the form

$$
\begin{aligned}
& V_{\beta}^{j}\left(\phi, \phi_{1}, T_{2}, T_{4}\right) \\
& \quad=\frac{1}{2} v^{\prime} t^{\prime} \xi_{1}^{j}\left(-2 i T_{p} \pi\right)\left(\alpha^{\prime}\right)^{n+m+3}\left(a_{n, m}+b_{n, m}\right) \\
& \quad\left(\left(k_{2} \cdot k_{1}\right)^{n}\left(k_{1} \cdot k_{4}\right)^{m}+\left(k_{2} \cdot k_{1}\right)^{m}\left(k_{4} \cdot k_{1}\right)^{n}\right. \\
& +\left(k_{2} \cdot k_{1}\right)^{n}\left(k \cdot k_{2}\right)^{m}+\left(k_{2} \cdot k_{1}\right)^{m}\left(k \cdot k_{2}\right)^{n} \\
& +\left(k_{1} \cdot k_{4}\right)^{m}\left(k \cdot k_{4}\right)^{n}+\left(k_{1} \cdot k_{4}\right)^{n}\left(k \cdot k_{4}\right)^{m} \\
& \left.+\left(k \cdot k_{2}\right)^{m}\left(k \cdot k_{4}\right)^{n}+\left(k \cdot k_{2}\right)^{n}\left(k \cdot k_{4}\right)^{m}\right) \operatorname{Tr}\left(\lambda_{4} \lambda_{1} \lambda_{2} \lambda_{\beta}\right)
\end{aligned}
$$

where $k$ is the momentum of the off-shell scalar field. Substituting the above vertex in the EFT amplitude (30), we produce all infinite scalar poles as

$$
\begin{aligned}
& 8 i \mu_{p}^{\prime} \beta^{\prime} \frac{\epsilon^{a_{0} \ldots a_{p}} \xi_{1 i} H_{a_{0} \ldots a_{p-1}}^{i} k_{3 a_{p}}}{p !\left(v^{\prime}+t^{\prime}+r\right)} \operatorname{Tr}\left(\lambda_{1} \lambda_{2} \lambda_{3} \lambda_{4}\right) \\
& \sum_{n, m=0}^{\infty}\left(a_{n, m}+b_{n, m}\right)\left[v^{\prime m} t^{\prime n}+v^{\prime n} t^{\prime m}\right] v^{\prime} t^{\prime} .
\end{aligned}
$$

Eventually, the S-matrix suggests that the string amplitude has just a double pole for the $p+1=n$ case. It emerges from the following Feynman diagram in EFT: ${ }^{3}$

$V\left(C_{p}, \phi_{1}, T\right) G(T) V_{a}\left(T, T_{4}, A\right) G_{a b}(A) V_{b}\left(A, T_{2}, T_{3}\right)$

\footnotetext{
${ }^{3}$ We suppress all gauge indices.
} 
with the derived vertices taken from the lower order effective actions,

$V\left(C_{p}, \phi_{1}, T\right)=\beta^{\prime} \mu_{p}^{\prime}\left(2 \pi \alpha^{\prime}\right)^{2} \frac{1}{(p+1) !} p^{i} \epsilon^{a_{0} \ldots a_{p}} H_{a_{0} \ldots a_{p}} \xi_{1 i}$,

$V_{a}\left(T, T_{4}, A\right)=T_{p}\left(2 \pi \alpha^{\prime}\right)\left(k_{4 a}+k_{a}\right)$,

$G_{a b}(A)=\frac{i \delta^{a b}}{\left(2 \pi \alpha^{\prime}\right)^{2} T_{p} u}$

$V_{b}\left(A, T_{2}, T_{3}\right)=T_{p}\left(2 \pi \alpha^{\prime}\right)\left(k_{2}-k_{3}\right)_{b}$;

$G(T)=\frac{i}{\left(2 \pi \alpha^{\prime}\right) T_{p}(u+r+w+1)}$

and $k$ is the off-shell tachyon's momentum. Replacing the above vertices by (36) we would reproduce its double pole too.

Note that by direct calculations, the presence of some new couplings such as $F^{(1)} \cdot F^{(2)}$ or $D \phi^{i(1)} \cdot D \phi_{i(2)}$ has been confirmed in the world volume of D-brane-anti D-brane systems $[19,20,56]$. Indeed making a string calculation we could produce all massless and tachyon singularities of the amplitudes.

While WZ coupling $C_{p} \wedge \mathrm{DT} \phi$ will not receive any higher derivative correction, and all the kinetic terms are fixed, they do not get any corrections either. Thus all other tachyon singularities give us clues about the structures of all order higher derivative corrections to various couplings and in this paper we could consistently fix their coefficients for good.

Note that all these couplings are found in the limit $p_{a} p^{a} \rightarrow 1 / 4$, thus we cannot compare these couplings with BSFT couplings. However, the tachyon's potential remains the same as in $\operatorname{BSFT}\left(V(T)=\mathrm{e}^{\pi \alpha^{\prime} m^{2} T^{2}}[58]\right)$, which is

$V\left(T^{i} T^{i}\right)=1+\pi \alpha^{\prime} m^{2} T^{i} T^{i}+\frac{1}{2}\left(\pi \alpha^{\prime} m^{2} T^{i} T^{i}\right)^{2}+\cdots$

where $m^{2}=-1 /\left(2 \alpha^{\prime}\right)$ is the tachyon's mass. Tachyon condensation is going to be carried out at $T \rightarrow \infty$; therefore, its potential will be sent to zero.

We think these corrections play a crucial role in determining singularities of the higher point functions of string theories. The Veneziano amplitude [69] was generalized in [21], and we hope to be able to address a supersymmetric generalization of the D-brane-anti-D-brane system by directly carrying out fermionic amplitudes [70]. We also hope to make progress on the generalization of the non-supersymmetric DBI and WZ effective actions in the near future.

Acknowledgements Some parts of the paper were written at Vienna University of Technology and at Queen Mary University of London. EH would like to thank K. Narain, L. Alvarez-Gaume for discussions and supports, also thanks to Mathematical Institute at Charles university for the hospitality. He is also grateful to IHES, CERN, UC Berkeley and Caltech for the warm hospitality. We thank A. Sagnotti, J. Polchinski, B. Jurco, O. Lechtenfeld, N. Arkani-Hamed, P. Horava, P. Sulkowski, G. Veneziano, J. Schwarz and W. Siegel for their insights and fruitful discussions. This work was supported by an ERC Starting Grant no. 335739 "Quantum fields and knot homologies", funded by the Euro- pean Research Council under the European Union's 7th Framework Programme. EH was also supported in part by Scuola Normale Superiore and by INFN.

Data Availability Statement This manuscript has no associated data or the data will not be deposited. [Authors' comment: We have not used any data as our paper is related to pure algebraic calculations.]

Open Access This article is distributed under the terms of the Creative Commons Attribution 4.0 International License (http://creativecomm ons.org/licenses/by/4.0/), which permits unrestricted use, distribution, and reproduction in any medium, provided you give appropriate credit to the original author(s) and the source, provide a link to the Creative Commons license, and indicate if changes were made.

Funded by SCOAP . $^{3}$.

\section{References}

1. M. Gutperle, A. Strominger, JHEP 0204, 018 (2002). arXiv:hep-th/0202210

2. A. Sen, JHEP 0204, 048 (2002). arXiv:hep-th/0203211

3. A. Sen, JHEP 0210, 003 (2002). arXiv:hep-th/0207105

4. A. Strominger, arXiv:hep-th/0209090;

5. F. Larsen, A. Naqvi, S. Terashima, JHEP 0302, 039 (2003). arXiv:hep-th/0212248

6. M. Gutperle, A. Strominger, Phys. Rev. D 67, 126002 (2003). arXiv:hep-th/0301038

7. N.D. Lambert, H. Liu, J.M. Maldacena, JHEP 0703, 014 (2007) arXiv:hep-th/0303139

8. A. Sen, Tachyon dynamics in open string theory. Int. J. Mod. Phys. A 20, 5513 (2005). arXiv:hep-th/0410103

9. A. Sen, arXiv:hep-th/9904207

10. D. Friedan, E.J. Martinec, S.H. Shenker, Nucl. Phys. B 271, 93 (1986)

11. T. Sakai, S. Sugimoto, Prog. Theor. Phys. 113, 843 (2005). arXiv:hep-th/0412141

12. T. Sakai, S. Sugimoto, Prog. Theor. Phys. 114, 1083 (2005). arXiv:hep-th/0507073

13. R. Casero, E. Kiritsis, A. Paredes, arXiv:hep-th/0702155

14. A. Dhar, P. Nag,. arXiv:0708.3233 [hep-th]

15. A. Sen, JHEP 9910, 008 (1999). arXiv:hep-th/9909062

16. E.A. Bergshoeff, M. de Roo, T.C. de Wit, E. Eyras, S. Panda, JHEP 0005, 009 (2000). arXiv:hep-th/0003221

17. A. Sen, Tachyon matter. JHEP 0207, 065 (2002). arXiv:hep-th/0203265

18. A. Sen, Mod. Phys. Lett. A 17, 1797 (2002). arXiv:hep-th/0204143

19. E. Hatefi, JCAP 1604(04), 055 (2016). arXiv:1601.06667 [hep-th]

20. E. Hatefi, JHEP 0903, 008 (2009). arXiv:0812.4216 [hep-th]

21. E. Hatefi, JHEP 1709, 025 (2017). arXiv:1707.06609 [hep-th]

22. A. Sen, JHEP 9808, 012 (1998). arXiv:hep-th/9805170

23. B. Michel, E. Mintun, J. Polchinski, A. Puhm, P. Saad, JHEP 1509, 021 (2015). arXiv:1412.5702 [hep-th]

24. O. Bergman, M.R. Gaberdiel, Phys. Lett. B 441, 133 (1998). arXiv:hep-th/9806155

25. A. Sen, JHEP 9809, 023 (1998). arXiv:hep-th/9808141

26. E. Dudas, J. Mourad, A. Sagnotti, Nucl. Phys. B 620, 109 (2002). arXiv:hep-th/0107081

27. E. Eyras, S. Panda, Nucl. Phys. B 584, 251 (2000). arXiv:hep-th/0003033

28. E. Eyras, S. Panda, JHEP 0105, 056 (2001). arXiv:hep-th/0009224

29. E. Hatefi, Phys. Lett. B 760, 509 (2016). arXiv:1511.04971 [hep-th]

30. A. Lerda, R. Russo, Int. J. Mod. Phys. A 15, 771 (2000). arXiv:hep-th/9905006 
31. V. Calo, G. Tallarita, S. Thomas, JHEP 1011, 063 (2010). arXiv:1003.6063 [hep-th]

32. G.R. Dvali, S.H.H. Tye, Brane inflation. Phys. Lett. B 450, 72 (1999). arXiv:hep-ph/9812483

33. D. Choudhury, JCAP 0307, 009 (2003). arXiv:hep-th/0305104

34. S. Kachru, Towards inflation in string theory. JCAP 0310, 013 (2003). arXiv:hep-th/0308055

35. A. Sagnotti, in Cargese '87, ed. by G. Mack et al. NonPerturbative Quantum Field Theory (Pergamon Press, 1988), p. 521. arXiv:hep-th/0208020

36. G. Pradisi, A. Sagnotti, Phys. Lett. B 216, 59 (1989)

37. P. Horava, Nucl. Phys. B 327, 461 (1989)

38. P. Horava, Phys. Lett. B 231, 251 (1989)

39. M. Bianchi, A. Sagnotti, Phys. Lett. B 247, 517 (1990)

40. M. Bianchi, A. Sagnotti, Nucl. Phys. B 361, 519 (1991)

41. A. Sagnotti, Phys. Lett. B 294, 196 (1992). arXiv:hep-th/9210127

42. E. Dudas, Class. Quant. Grav. 17, R41 (2000). arXiv:hep-ph/0006190

43. C. Angelantonj, A. Sagnotti, Phys. Rept. 371, 1 (2002). arXiv:hep-th/0204089 [Erratum-ibid. 376339 (2003)]

44. E. Hatefi, JHEP 1307, 002 (2013). arXiv:1304.3711 [hep-th]

45. E. Hatefi, A.J. Nurmagambetov, I.Y. Park, JHEP 1304, 170 (2013). arXiv:1210.3825 [hep-th]

46. E. Hatefi, A.J. Nurmagambetov, I.Y. Park, Nucl. Phys. B 866, 58 (2013). arXiv:1204.2711 [hep-th]

47. J. Polchinski, Brane/antibrane dynamics and KKLT stability. arXiv:1509.05710 [hep-th]

48. S. de Alwis, R. Gupta, E. Hatefi, F. Quevedo, JHEP 1311, 179 (2013). arXiv:1308.1222 [hep-th]

49. J. Polchinski, Phys. Rev. Lett. 75, 4724 (1995). arXiv:hep-th/9510017

50. E. Witten, Nucl. Phys. B 460, 335 (1996). arXiv:hep-th/9510135

51. E. Hatefi, JHEP 1005, 080 (2010). arXiv:1003.0314 [hep-th]

52. R.C. Myers, Dielectric branes. JHEP 9912, 022 (1999). arXiv:hep-th/9910053
53. C. Kennedy, A. Wilkins, Phys. Lett. B 464, 206 (1999). arXiv:hep-th/9905195

54. E. Hatefi, Selection rules and RR couplings on non-BPS branes. JHEP 1311, 204 (2013). arXiv:1307.3520

55. A. Sen, Phys. Rev. D 68, 066008 (2003). arXiv:hep-th/0303057

56. M.R. Garousi, E. Hatefi, Nucl. Phys. B 800, 502 (2008). arXiv:0710.5875 [hep-th]

57. E. Hatefi, On D-brane anti D-brane effective actions and their corrections to all orders in alpha-prime. JCAP 1309, 011 (2013). arXiv:1211.5538 [hep-th]

58. D. Kutasov, M. Marino, G.W. Moore, arXiv:hep-th/0010108

59. P. Kraus, F. Larsen, Phys. Rev. D 63, 106004 (2001). arXiv:hep-th/0012198

60. T. Takayanagi, S. Terashima, T. Uesugi, JHEP 0103, 019 (2001). arXiv:hep-th/0012210

61. D. Quillen, Superconnections and the chern character. Topology 24, 89-95 (1995)

62. N. Berline, E. Getzler, M. Vergne, Heat Kernels and Dirac Operators (Springer, Heidelberg, 1991)

63. G. Roepstorff, J. Math. Phys. 40, 2698 (1999). arXiv:hep-th/9801040

64. E. Hatefi, On non-BPS effective actions of string theory. Eur. Phys. J. C 78(5), 383 (2018). arXiv:1703.09996 [hep-th]

65. H. Liu, J. Michelson, Nucl. Phys. B 614, 330 (2001). arXiv:hep-th/0107172

66. E. Hatefi, Phys. Rev. D 86, 046003 (2012). arXiv:1203.1329 [hepth]

67. A. Fotopoulos, JHEP 0109, 005 (2001). arXiv:hep-th/0104146

68. J.H. Schwarz, Highly effective actions. JHEP 1401, 088 (2014). arXiv:1311.0305 [hep-th]

69. G. Veneziano, Nuovo Cim. A 57, 190 (1968)

70. E. Hatefi, More on D-brane effective actions with their all order corrections (to appear in 2019) 